\title{
Modelo de carroceria e seu impacto sobre o bem-estar e a qualidade da carne dos suínos
}

\author{
Impact of the truck design on welfare and meat quality in pigs
}

\author{
Osmar Antonio Dalla Costa ${ }^{{ }^{*}}$ Jorge Vitor Ludke ${ }^{\text {II }}$ Mateus José Rodrigues Paranhos da Costa ${ }^{\text {III }}$ \\ Luigi Faucitano $^{\text {IV }}$ José Vicente PelosoV $^{V}$ Darlan Dalla Roza $^{\text {VI }}$
}

\section{RESUMO}

O objetivo deste trabalho foi avaliar o efeito do modelo de carroceria (MC) (metálica dupla ou simples e simples de madeira) sobre a freqüencia de diferentes tipos de lesões na carcaça (FLC) e sobre a qualidade da carne dos suínos. Foram utilizadas 360 fêmeas com peso vivo médio de 132,72ะ11,09kg. Para a análise estatística, considerou-se no modelo os efeitos de bloco (BL), o MC e a interação entre MC x BL. Verificou-se um efeito do MC sobre a freqüencia de lesões na carcaça provenientes de briga e a freqüência total de lesões, sobre o $\mathrm{pH}_{1}$ do músculo Semispinalis capitis (SC), sobre o $\mathrm{pH}_{U}$ dos músculos SC, Longissimus dorsi (LD) e Semimembranosus (SM) e sobre a cor dos músculos LD e SM. Suínos transportados em carroceria metálica simples apresentaram maiores valores de $\mathrm{pH}$ e da cor dos músculos avaliados. Conclui-se que suínos transportados em carroceria simples apresentam maior prevalência de lesões na carcaça e que o transporte dos suínos em carroceria metálica teve efeito positivo sobre os valores de pH e da cor carne.

Palavras-chave: carroceria dupla, carroceria simples, carroceria de madeira, carroceria metálica, escore de lesão na carcaça, manejo préabate, transporte de suínos.

\section{ABSTRACT}

This experiment was aimed at evaluating the effect of three truck-tier models (MC) (metallic simple tier, metallic duple tier and wood simple tier) on the frequency of different kinds of carcass lesions (FLC) and pork quality. For this study, three hundred and sixty gilts with an average body weight of $132.72 \pm 11.09 \mathrm{~kg}$ were used. Statistic analysis of the data considered the effects of Blocks (BL), MC and the interaction $M C \times B L . M C$ had significant effect on frequency of lesions caused by fighting, on total frequency of injuries, on $\mathrm{pH}_{1}$ of muscle Semispinalis capitis (SC), on $\mathrm{pH}_{U}$ of muscle SC, Longissimus dorsi (LD) and Semimembranosus (SM) and on color of the muscles $L D$ e SM. Pigs transported in simple metallic truck-tier showed increased values of $\mathrm{pH}$ and muscles color. As a conclusion, transportation of slaughter pigs in simple wood truck-tier affects animal welfare negatively, while metallic truck-tier showed positive effects on meat $p H$ and color compared to.

Key words: carcass blemishes score, duple truck-tier, metallic truck-tier, pre-slaughter handling, simple trucktier, swine transport, wood truck-tier.

\section{INTRODUÇÃO}

Durante os procedimentos do manejo préabate, os suínos são expostos a diferentes agentes estressantes que influenciam a qualidade da carne (COOK, 1999). Portanto, o manejo pré-abate, embora

IPrograma de Pós-graduação em Zootecnia (Produção Animal), Faculdade de Ciências Agrárias e Veterinárias (FCAV), Universidade Estadual Paulista (UNESP). Embrapa Suínos e Aves, BR 153, Km 110, CP 21, 89700-000, Concórdia, SC, Brasil. E-mail: osmar@cnpsa.embrapa.br. Autor para correspondência.

"Embrapa Suínos e Aves, Concórdia, SC, Brasil.

IIIDepartamento de Zootecnia, Grupo de Estudos e Pesquisas em Etiologia e Ecologia Animal (ETCO), FCAV, UNESP, Jaboticabal, SP, Brasil.



v'Sadia S. A., Concórdia, SC, Brasil.

VITRIEL-HT Industria de Equipamentos Rodoviários Ltda, Erechim, RS, Brasil. 
de curta duração, pode ocasionar perdas quantitativas e qualitativas na produção de carne suína. O transporte dos suínos da granja ao frigorífico é considerado a etapa mais estressante, devido à interação homem suíno (deslocamento dos animais, embarque, desembarque) e devido à utilização indevida do choque elétrico (FAUCITANO, 2001).

Estudos mostraram os efeitos de condições do transporte, como a densidade do carregamento e o tempo e a distância do transporte (PEREZ et al., 2002; WARRISS, 1998), em perdas econômicas relacionando estes fatores à taxa de mortalidade, às lesões da carcaça e aos efeitos sobre a qualidade da carne suína. Entretanto, poucos estudos focalizaram os efeitos do modelo de carroceria sobre o bem-estar e a qualidade da carne dos suínos. Ainda assim, a maior parte das avaliações descritas tem-se restringido somente a algumas características do veículo, como o dispositivo de carregamento (GUÁRDIA et al., 2004; 2005; NANNI COSTA et al., 1999), o tipo de piso (GUÁRDIA et al., 2004/2005), o número das plataformas (LAMBOOJ \& ENGEL, 1991; GUISE \& PENNY, 1992; BARTON-GADE et al., 1996), a ventilação e a altura da plataforma (CHRISTENSEN, 2003).

As condições do transporte podem afetar o bem-estar e a qualidade da carne dos suínos. BARTONGADE et al. (1996) relataram um efeito significativo do compartimento do transporte, especialmente nos suínos transportados na parte dianteira e na parte traseira, sobre a qualidade da carne e as lesões da pele. Os efeitos na qualidade da carne eram possivelmente causados pelo estresse físico originado pela necessidade de o animal manter a posição ereta a fim de lidar com as vibrações, que são mais elevadas nessas posições (BARTON-GADE et al., 1996; RANDALL et al., 1996).

No Brasil, a preocupação com as condições do transporte dos suínos (distância, sistema de condução, modelo de carroceria) sobre o bem-estar e a qualidade da carne tem sido muito restrita. Em condições brasileiras, CULAU et al. (1991) e LUDTKE et al. (2004) não encontraram efeito significativo das condições do transporte sobre a qualidade do bemestar animal e da carne suína. Todavia, DALLA COSTA (2005) verificou que suínos transportados em carrocerias simples (um piso) apresentaram uma freqüência de lesões na carcaça significativamente maior em relação aos transportados em carrocerias duplas (dois pisos), e esses animais apresentaram valores de $\mathrm{pH}_{\mathrm{U}}$ dos músculos Longissimus dorsi e Semimembranosus significativamente menores em comparação aos transportados em carrocerias simples.

O objetivo deste estudo foi avaliar os efeitos do modelo de carroceria (metálica dupla e simples e simples de madeira) sobre as lesões na carcaça e sobre a qualidade da carne dos suínos.

\section{MATERIAL E MÉTODOS}

Foram utilizadas 360 fêmeas oriundas de cruzamentos industriais com peso vivo médio de $132,72 \pm 11,09 \mathrm{~kg}$ e um período de alojamento médio de 144 dias, para as fases de crescimento e terminação. Os animais no experimento foram identificados na orelha, com brincos plásticos, e tatuados na paleta e no pernil em um período 48 horas antes do carregamento. Foram utilizadas quatro granjas em sistemas de terminações de suínos, duas no verão (fevereiro de 2002, com temperaturas mínima e máxima absolutas de 13,0 e 33,50, respectivamente, médias das temperaturas mínimas e máximas de 19,9 e $27,2^{\circ}$, respectivamente) e outras duas no inverno (julho de 2002, com temperaturas mínima e máxima absolutas de 0,0 e $29,0^{\circ}$, respectivamente, e as médias das temperaturas mínimas e máximas de 12,8 e $18,8^{\circ}$, respectivamente). As granjas tinham capacidade média de alojar 500 suínos. Nas granjas avaliadas, foram escolhidas aleatoriamente 4 baias/modelo de carroceria (6 animais baia ${ }^{-1}$ ), totalizando 12 baias granja ${ }^{-1}$ para a realização do experimento. Durante o manejo pré-abate, na granja, antes do carregamento, os suínos receberam um tempo de jejum de 12 horas. As granjas utilizadas no experimento estavam a uma distância média de $35 \mathrm{~km}$ do frigorífico, o que correspondeu ao tempo de transporte de 65 minutos, em média.

O transporte dos suínos foi realizado em 3 modelos de carrocerias, duas metálicas (simples e dupla), desenvolvidos pela empresa TRIEL-HT, e a outra de madeira simples. Na carroceria dupla metálica, foram transportados 96 animais em 16 boxes que estavam sobre um chassi Volkswagen trucado modelo 24.220 (6x4), ano 2000. Na carroceria simples TRIELHT, foram transportados 44 suínos em quatro boxes, e esta estava sobre chassi Mercedes Bens modelo 1316 (4x2), ano 1981. Na carroceria simples de madeira, foram transportados 35 suínos, em três boxes, e estava sobre um chassi Mercedes Benz modelo 1111 (4x2) ano 1978.

No frigorífico, os suínos foram desembarcados com o auxílio de uma plataforma móvel e conduzidos até as baias de descanso coletivas. No desembarque e deslocamento dos animais até as baias de descanso, estes foram misturados aleatoriamente dentro de cada grupo de modelo de carroceria. No frigorífico, os suínos permaneceram em descanso por 3 horas.

A insensibilização (ou eletronarcose) foi aplicada automaticamente, transferindo alta voltagem (700V) e amperagem acima de 1,25amps (Valhalla, Stork 
RMS b.v., Lichtenvoorde, Holanda). Após a insensibilização, os animais foram imediatamente sangrados na posição horizontal e suspensos ao fim da mesa de sangria na nórea contínua da linha de abate.

A freqüência de lesões de agressão/ escoriação na pele foi realizada na meia-carcaça esquerda dos suínos, através de avaliação visual, pela contagem do número de lesões no lado esquerdo dos animais, vinte e quatro horas após abate, quando registrou-se a freqüência de lesões por carcaça e a origem das lesões, classificando-as em: a) manejo, b) densidade; c) briga, sendo que o índice do escore de lesões seguindo a metodologia descrita por ITP (1996). Para realização da análise da variância, as freqüências de lesão foram transformadas para $\sqrt{y+1}$.

As carcaças dos suínos permaneceram em câmara fria, em temperaturas variando entre 1 e $4^{\circ} \mathrm{C}$, por 24 horas. As medidas do $\mathrm{pH}$ foram realizadas na meia carcaça esquerda nos músculos Semispinalis capitis (SC), Longissimus dorsi (LD) e Semimembranosus (SM), 45 minutos $\left(\mathrm{pH}_{1}\right)$ e 24 horas após o abate $\left(\mathrm{pH}_{\mathrm{U}}\right)$. Na avaliação do $\mathrm{pH}$, foi utilizado o pHmetro portátil da marca Mettler Toledo (MP 120 pH Meter, Suíça) com eletrodo DXK-S7/25 protegido para inserção no músculo.

A avaliação da cor dos músculos Longissimus dorsi (CM-LD) e Semimembranosus (CMSM), foi realizada 24 horas após o abate através da unidade de dispersão e reflexão de luz com a utilização do aparelho fotômetro específico (Opto Star ${ }^{\mathrm{TM}}$ SFK).

A porcentagem de perda de água por gotejamento (\%PG) do músculo Semimembranosus foi determinada em $50 \%$ dos animais que participaram deste estudo, com a coleta de amostras de aproximadamente $110 \mathrm{~g}$ desse músculo, livres de gordura, que, após a pesagem, foram suspensas em redes de nylon seladas dentro de sacos plásticos, e assim permaneceram na câmara fria submetidas a temperaturas variando entre 1 e $4^{\circ} \mathrm{C}$ por 48 horas. A porcentagem de perda de água por gotejamento foi calculada como resultado da diferença entre o peso inicial e o peso final da amostra dividido pelo peso inicial e multiplicado por 100 .
O presente estudo foi realizado em quatro granjas distintas, duas no verão e outras duas no inverno. Assim, os efeito de granja não foram levados em conta na discussão, porque o objetivo de incluir os efeitos no modelo foi apenas para melhorar o ajuste dos dados, pois a estação do ano apenas restringiu a aleatorização, como um efeito de bloco.

As análises de variância foram realizadas por meio do método de quadrados mínimos, pelo procedimento GLM do programa SAS (2001), utilizando-se no modelo os efeitos de bloco (inverno e verão), o modelo de carroceria (MC=simples Triel-HT, dupla Triel-HT, e simples de madeira) e a interação entre bloco e modelo de carroceria (BL versus MC).

\section{RESULTADOS E DISCUSSÃO}

As médias ajustadas e os desvios-padrão para as freqüências de lesões na carcaça provenientes de manejo (FLC-M), de densidade (FLC-D), de brigas (FLC-B) e de lesões totais (FLC-T), avaliadas 24 horas após o abate, em função de estação do ano, do modelo de carroceria e da posição do animal dentro da carroceria do caminhão, são apresentadas na tabela 1 . Não foram observados efeitos do modelo de carroceria sobre a FLC- M ( $\mathrm{P}=0,6608)$ e FLC-D ( $\mathrm{P}=07054)$. Todavia, o modelo de carroceria influenciou significativamente FLC-B $(\mathrm{P}=0,0026)$ e FLC-T $(\mathrm{P}=0,0294)$.

O transporte dos suínos em carroceria simples proporcionou maior número de lesões nas carcaças provocadas por briga e no total das lesões, em relação aos animais transportados nos modelos carroceria Triel-HT simples e dupla, e esses não diferiram ( $\mathrm{P}>0,05)$ ente si. DALLA COSTA (2005), ao avaliar os modelos de carrocerias Triel-HT dupla e simples, encontrou uma maior incidência de lesões na carcaça provenientes por briga e de lesões totais nos suínos transportados em carroceria metálica simples, em relação aos transportados em carrocerias duplas.

Na figura 1, estão apresentados os valores dos $\mathrm{pH}_{1}$ e do $\mathrm{pH}_{\mathrm{U}}$ dos músculos SC, LDe SM, e da cor dos músculos LD e SM e da porcentagem de perda água do músculo SM por modelo de carroceria. O

Tabela 1 - Médias ajustadas e desvios-padrão para as freqüências de lesões na carcaça provenientes de manejo (FLC-M), de densidade (FLCD), de brigas (FLC-B) e de lesões totais (FLC-T), avaliadas 24 horas após o abate, em função do modelo de carroceria.

\begin{tabular}{|c|c|c|c|c|}
\hline Modelo de carroceria & FLC-M & FLC-D & FLC-B & FLC-T \\
\hline Simples Triel & $0,99 \pm 1,22$ & $1,03 \pm 1,63$ & $4,27 \pm 5,36^{b}$ & $6,29 \pm 6,10^{b}$ \\
\hline Dupla Triel & $0,95 \pm 1,24$ & $1,01 \pm 1,66$ & $4,44 \pm 5,13^{\mathrm{b}}$ & $6,40 \pm 6,13^{b}$ \\
\hline Simples & $0,87 \pm 1,15$ & $0,97 \pm 2,08$ & $6,47 \pm 8,30^{\mathrm{a}}$ & $8,33 \pm 8,67^{\mathrm{a}}$ \\
\hline
\end{tabular}

Na coluna, as médias seguidas de letras minúsculas distintas diferem significativamente pelo teste $\mathrm{T}(\mathrm{P}<0,05)$.

Ciência Rural, v.37, n.5, set-out, 2007. 


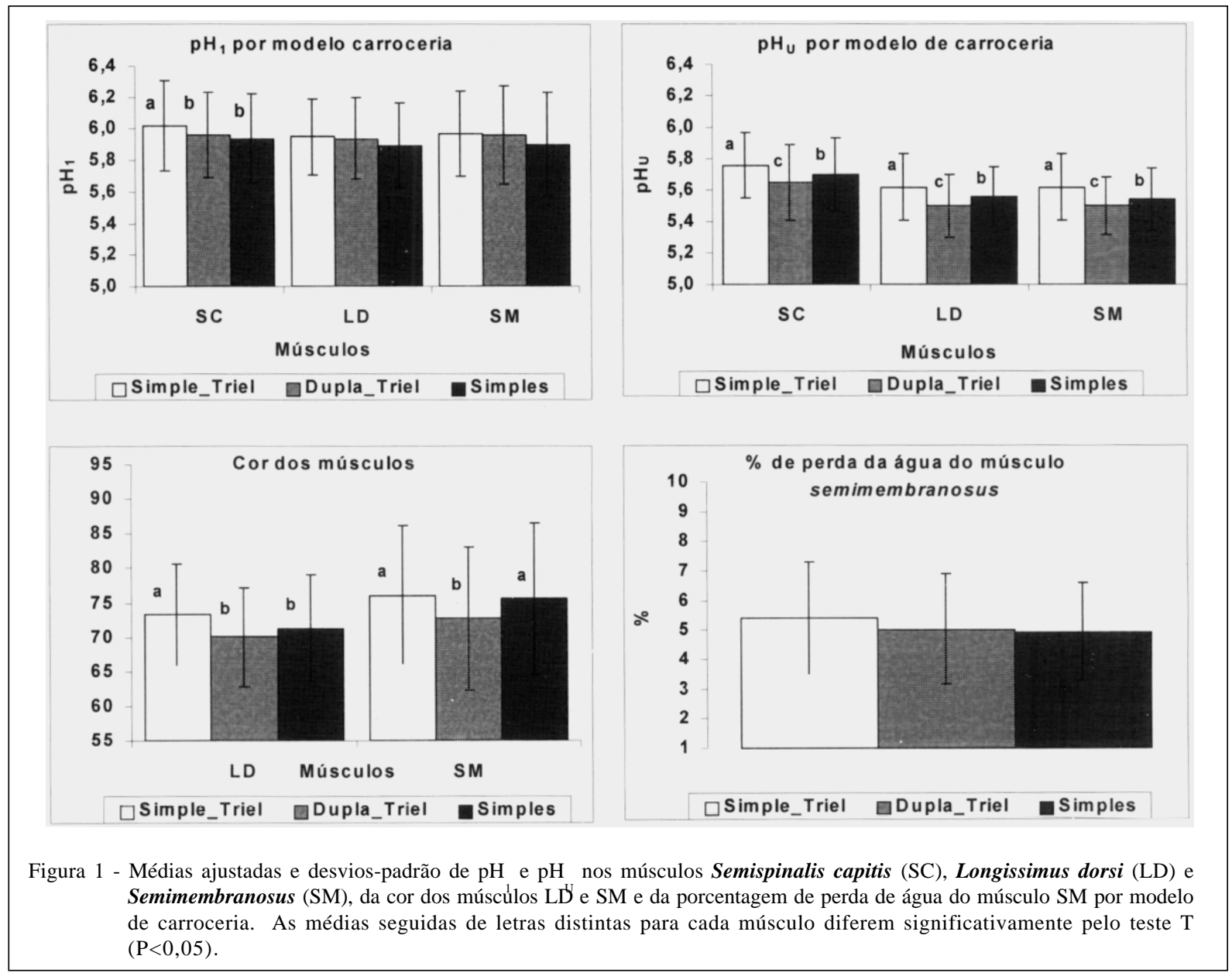

modelo de carroceria não influenciou significativamente o $\mathrm{pH}_{1}$ dos músculos $\mathrm{LD}(\mathrm{P}=0,073)$ e SM ( $\mathrm{P}=0,065)$. Todavia, o modelo de carroceria influenciou significativamente o $\mathrm{pH}_{1}$ do músculo $\mathrm{SC}$ $(\mathrm{P}=0,020)$ e dos $\mathrm{pH}_{\mathrm{U}}$ dos músculos $\mathrm{SC}, \mathrm{LD} \mathrm{SM}$ $(\mathrm{P}=0,0001)$, além da cor dos músculos $\mathrm{LD}(\mathrm{P}=0,003)$ e do $\mathrm{SM}(\mathrm{P}=0,008)$.

Suínos transportados nos modelos de carroceria metálica simples apresentaram maiores valores de $\mathrm{pH}_{1}$ do músculo $\mathrm{SC}$, diferindo significativamente dos animais que foram transportados em carrocerias simples de madeira, os quais apresentaram menores valores de $\mathrm{pH}_{1}$ do músculo SC. Esses resultados diferem dos obtidos por DALLA COSTA et al. (2006), que não observaram efeito dos modelos de carrocerias metálicas Triel-HT (simples e dupla) sobre $\mathrm{pH}_{1}$ do músculo SC.

Suínos transportados em carrocerias metálicas modelo Triel-HT simples apresentaram os maiores valores de $\mathrm{pH}_{\mathrm{U}}$ dos músculos SC, LD e SM, enquanto que os animais transportados nas carrocerias metálicas modelo Triel-HT dupla apresentaram os menores valores de $\mathrm{pH}_{U}$, e os suínos transportados em carrocerias simples de madeira apresentaram valores intermediários do $\mathrm{pH}_{\mathrm{U}}$.

Apesar do efeito significativo do modelo de carroceria sobre o $\mathrm{pH}_{1}$ do músculo $\mathrm{SC}$ e do $\mathrm{pH}_{\mathrm{U}}$ dos músculos SC, LDe SM, esta diferença representa muito pouco biologicamente sobre a qualidade da carne. Os resultados obtidos no presente estudo estão de acordo com os obtidos por DALLA COSTA et al. (2006), que, avaliando os modelos de carrocerias metálicas simples e duplas, também observaram que os suínos transportados em carrocerias simples apresentaram valores significativamente maiores em relação aos transportados em carrocerias metálicas duplas. Estudos conduzidos por GUÁRDIA et al. (2004) verificaram que suínos transportados em carrocerias com piso de alumínio ou ferro apresentam maior incidência de PSE em relação aos transportados em piso de poliéster. Contudo, os resultados do presente estudo diferiram dos encontrados por LUDTKE et al. (2004), que não observaram efeito significativo do modelo de carroceria (piso móvel, sistema de aspersão de água e coleta de dejetos e veículo convencional de piso fixo, rampa adaptável sem sistema de coleta de dejetos) e do 
sistema de condução (pranchas de alumínio e bastão elétrico) sobre a qualidade da carne dos suínos ( $\mathrm{pH}$ e $\mathrm{pH}$ do músculo SM, capacidade de retenção de ágúa do Uúsculo SM e da cor e porcentagem de carcaças com problema de PSE do músculo LD).

O modelo de carroceira influenciou a cor dos músculos $\mathrm{LD}(\mathrm{P}=0,002)$ e $\mathrm{SM}(\mathrm{P}=0,011)$, sendo que os suínos transportados sobre a carroceria metálica simples apresentaram maiores valores da cor dos músculos LD e os animais transportados na carroceria metálica dupla e simples de madeira e os suínos transportados em carroceria dupla aprestaram maiores valores da cor do músculo SM em relação aos transportados em carrocerias simples. Os resultados obtidos no presente estudo diferem dos obtidos por DALLA COSTA et al. (2006) e LUDTKE et al. (2004), que não observaram efeito do modelo de carroceria (simples e dupla) na cor dos músculos avaliados e dos encontrados por LUDTKE et al. (2004).

O modelo de carroceria não influenciou $(\mathrm{P}=0,313)$ a porcentagem de perda de água do músculo SM. Esses resultados validam os obtidos por LUDTKE et al. (2004), que também não encontraram efeito significativo do manejo pré-abate sobre a perda de água por gotejamento.

\section{CONCLUSÕES}

Transportar suínos em carrocerias simples aumenta as lesões na carcaça. O transporte dos suínos em carrocerias simples metálicas aumenta os valores do $\mathrm{pH}$ e da cor dos músculos.

\section{REFERÊNCIAS}

BARTON-GADE, P.A. et al. Effect of tier ventilation during transport on blood parameters and meat quality in slaughter pigs. In: EU-SEMINAR: NEW INFORMATION ON WELFARE AND MEAT QUALITY OF PIGS AS RELATED TO HANDLING, TRANSPORT AND LAIRAGE CONDITIONS, 1966, Mariensee. Proceedings... Kulmbach: Landbauforschung Völkenrode, 1996. V.166. p.101-116.

CHRISTENSEN, L. L'expérience danoise dans le transport des porcs, avec l'attention spéciale à la conception des véhicules de transport à étages. In: Cahiers du Colloque sur la Production Porcine. Saint-Hyacinthe, Canada: Centre de Référence en Agriculture et Agro-alimentaire de Québec, 2003. p.91-108.

COOK, J.C. Neurological measures to qualify welfare aspects of stunning. In: INTERNATIONAL WORKSHOP ON STUNNING SYSTEMS FOR PIGS AND ANIMAL WELFARE, 1999, Billund, Denmark. Proceedings... Billund: Danish Meat Research Institute, 1999. p.25-27.

CULAU, P.O.V.; et al. The effect of transportation distance and preslaughter lairage time on the pigmeat quality. In: INTERNATIONAL CONGRESS OF MEAT SCIENCE AND TECHNOLOGY, 37., 1991, Kulmbach, Germany. Proceedings... Kulmbach, 1991. p.224-228.
DALLA COSTA, O.A. Efeito de manejo pré-abate no bemestar e na qualidade de carne dos suínos. 2005. $160 \mathrm{f}$. Tese (Doutorado em Zootecnia) - Faculdade de Ciências Agrárias e Veterinária, Universidade Estadual Paulista, Jaboticabal.

DALLA COSTA, O.A. et al. Effects of the season of the year, truck type and location on truck on skin bruises and meat quality in pigs. Livestock Production Science, v.107, n.1, p.29-36, 2007.

FAUCITANO, L. Efeitos do manuseio pré-abate sobre o bemestar e sua influência sobre a qualidade da carne. In: CONFERENNCIA VIRTUAL INTERNACIONAL SOBRE QUALIDADE DA CARNE SUÍNA, 2000, Concórdia. Anais... Concórdia: Embrapa Suínos e Aves, 2001. p.55-75. (Embrapa Suínos e Aves. Documentos, 69).

GUÀRDIA, M.D. et al. A risk assessment of DFD meat due to pre-slaughter conditions in pigs. Meat Science, v.70, p.709716, 2005.

GUÀRDIA, M.D. et al. A risk assessment of PSE condition due to pre-slaughter conditions and RYR1 gene in pigs. Meat Science, v.67, p.471-478, 2004.

GUISE, H.J.; PENNY, R.H.C. Pig welfare from farm to factory. Is there a need for more research? Pig Veterinary Journal, v.30, p.16-22, 1992.

ITP - INSTITUT TECHNIQUE DU PORC. Notation des hématomes sur couenne - porcs vivant ou carcasses. Le Rheu: ITP, 1996. 45p.

LAMBOOIJ, E.; ENGEL, B. Transport of slaughter pigs by road over a long distance : some aspects of loading density and ventilation. Livestock Production Science, v.28, p.163174, 1991.

LUDTKE, C.B. et al. Avaliação da influência do transporte no bem-estar e qualidade da carne suína. In: CONGRESSO LATINO AMERICANO DE SUINOCULTURA, 2.; CONGRESSO DE SUINOCULTURA DO MERCOSUL, 4., 2004, Foz do Iguaçu. Anais... Paulínia: Animalword, 2004. p.279-280.

NANNI COSTA, L. et al. Influence of loading method and stocking density during transport on meat and dry-cured ham quality in pigs with different halothane genotypes. Meat Science, v.51, p.391-399, 1999.

PÉREZ, M.P. et al. Effect of transport time on welfare and meat quality in pigs. Meat Science, v.61, p.425-433, 2002.

RANDALL, J.M. et al. Vibration on pig transporters: implications for reducing stress. In: EU-SEMINAR: NEW INFORMATION ON WELFARE AND MEAT QUALITY OF PIGS AS RELATED TO HANDLING, TRANSPORT AND LAIRAGE CONDITIONS, 1996, Mariensee. Proceedings... Mariensee, Germany: Landbauforschung Völkenrode, 1996. p.143-159.

SAS INSTITUTE. System for Microsoft Windows: release 8.2. Cary, 2001. 1 CD-ROM.

WARRISS, P.D. Choosing appropriate space allowances for slaughter pigs transported by road: a review. Veterinary Record, v.142, p.449-454, 1998. 